\title{
Reconstruction of the Central Layer of the Human Cerebral Cortex from MR Images
}

\author{
Chenyang $\mathrm{Xu}^{1}$, Dzung L. Pham ${ }^{1,4}$, Jerry L. Prince ${ }^{1,2,3}$, \\ Maryam E. Etemad ${ }^{2}$, and Daphne N. Yu ${ }^{2}$ \\ ${ }^{1}$ Electrical and Computer Engineering, ${ }^{2}$ Biomedical Engineering, ${ }^{3}$ Radiology, \\ The Johns Hopkins University, Baltimore MD 21218, USA. \\ ${ }^{4}$ Laboratory of Personality \& Cognition, GRC/NIA/NIH, Baltimore, MD 21214, USA.
}

\begin{abstract}
Reconstruction of the human cerebral cortex from MR images is a fundamental step in human brain mapping and in applications such as surgical path planning. In a previous paper, we described a method for obtaining a surface representation of the central layer of the human cerebral cortex using fuzzy segmentation and a deformable surface model. This method, however, suffers from several problems. In this paper, we significantly improve upon the previous method by using a fuzzy segmentation algorithm robust to intensity inhomogeneities, and using a deformable surface model specifically designed for capturing convoluted sulci or gyri. We demonstrate the improvement over the previous method both qualitatively and quantitatively, and show the result of its application to six subjects. We also experimentally validate the convergence of the deformable surface initialization algorithm.
\end{abstract}

\section{Introduction}

Reconstruction of the human cerebral cortex has many research and clinical uses, including applications in human brain mapping, functional imaging, and neurosurgical path planning. It is a difficult problem because of imaging noise, partial volume averaging, image intensity inhomogeneities, extremely convoluted cortical structures, and the requirement to preserve anatomical topology. Preservation of topology is important for morphometric analysis, surgical path planning, and functional mapping where a representation consistent with anatomical structure is required. During the last decade, there has been a considerable amount of work in obtaining a surface representation of the cortex [1-6]. Most reported methods, however, either require extensive manual interaction [5] or are capable of reconstructing only the visible cortex [1-4].

In [6], a hybrid system combining fuzzy segmentation, isosurfaces, and deformable surface models was presented for reconstructing the central layer of the cortex from magnetic resonance (MR) images (see Sect. 2). This method suffers from several problems, however. First, if intensity inhomogeneities are present in the acquired images, the segmentation used may yield inaccurate results. Second, it was not known whether the iterative process used to initialize the deformable surface would converge in general since it was only applied to one subject. Third, the traditional deformable surface model

This work was partially supported by the NSF Presidential Faculty Fellow Award MIP9350336 and a Whitaker Foundation graduate fellowship. 
used in [6] has difficulties converging to boundary concavities, resulting in the loss of deeply convoluted sulci or gyri in the surface reconstruction.

In this paper, we present several improvements over the method presented in [6] for reconstructing the central layer of the entire human cerebral cortex. We address the problems of the previous method by using a fuzzy segmentation algorithm which is robust to intensity inhomogeneities, experimentally validating the convergence of the initialization algorithm, and using a deformable surface model specifically designed for capturing convoluted sulci or gyri. In Sect. 2, we briefly review the method reported in [6]. In Sect. 3, we describe the segmentation and deformable surface algorithms used in our new cortex reconstruction method. In Sect. 4, we present and discuss the results of applying our method to six different subjects.

\section{Background}

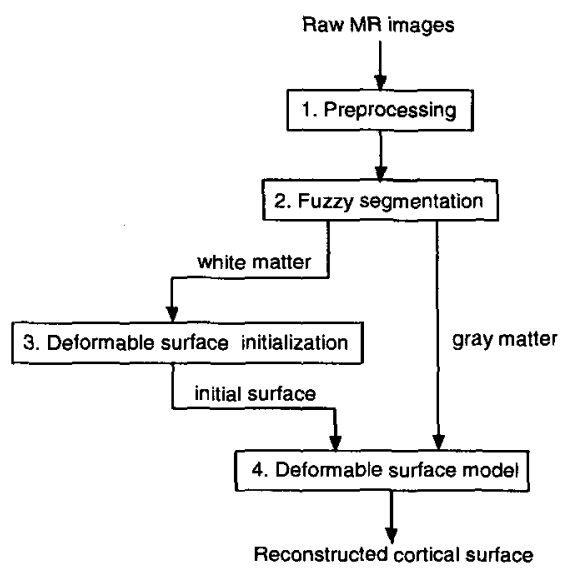

Fig. 1. Overview of cortical surface reconstruction method.
The method reported in [6] has four major steps as illustrated in Fig. 1. After the MR data (T1-weighted image volumes with voxel size $0.9375 \times 0.9375 \times 1.5 \mathrm{~mm}$ ) are acquired, the images are preprocessed to remove extracranial tissue, cerebellum, and brain stem. Each volume is then interpolated to isotropic voxels. Next, a fuzzy cmeans clustering algorithm (FCM) is used to segment the preprocessed data into three tissue classes: cerebrospinal fluid (CSF), gray matter (GM), and white matter (WM). As a result, each tissue is represented as a fuzzy membership function. The use of a fuzzy segmentation offers robustness to noise and partial volume averaging effects and removes the dependency on raw image intensity in later steps.

Once the images have been segmented, an initial estimate of the GM/WM interface is obtained before refinement to the final central layer. Obtaining an initial surface near the desired cortical surface is important because it provides a good starting position for deforming the surface, yields a proper initial parameterization of the deformable surface, significantly reduces the reconstruction time, and improves the reconstruction results. This is accomplished by applying the following operations to the WM membership function. First, hippocampal formations are manually removed, regions around the brain stem are manually filled, and ventricles are automatically filled in the WM membership function. An automatic iterative algorithm is then applied to the WM membership function to ensure that the resulting initial surface has a topology equivalent to a sphere. This algorithm consists of the following steps: 


\section{Compute isosurface on WM membership function at 0.5 .}

2. Extract largest connected mesh in the isosurface and remove singular points from the connected mesh.

3. Compute Euler characteristic $\chi$ on the extracted mesh. If $\chi<2$, median filter the WM membership function, recompute isosurface at 0.5 and go to Step 2. If $\chi=2$, then the surface topology is equivalent to a sphere, and the initialization is complete.

The final output of this process is a smoothed version of the GM/WM interface with the desired topology.

After the initialization is obtained, a traditional deformable surface model $[7,8]$ is used to refine the initial surface to the central layer of the gray matter.

\section{Methods}

In this section, we describe two major improvements upon the method described in Sect. 2. The standard FCM segmentation used in the previous method is replaced with an adaptive segmentation that is robust to intensity inhomogeneities. In addition, instead of using a traditional deformable surface model, an enhanced model is used that allows a more accurate reconstruction of the cortex.

\subsection{Adaptive Fuzzy C-Means Segmentation}

MR images may have intensity inhomogeneities caused by nonuniformities in the RF field during acquisition. The result is a slowly varying shading artifact over the image that can produce errors in intensity-based segmentation methods like FCM. These errors could potentially cause certain regions of the reconstructed cortex to shift into the WM or CSF. In [9], an adaptive fuzzy c-means algorithm (AFCM) was proposed for segmenting 2-D images that are corrupted by intensity inhomogeneities. Using an iterative algorithm, AFCM simultaneously computes fuzzy membership functions for each tissue class, the mean intensity of each tissue class (called the centroid), and an estimate of the inhomogeneity, which is modeled as a gain field. It was shown in [9] that AFCM achieves significantly lower error rates than FCM when segmenting images corrupted by intensity inhomogeneities. Thus, in order to make the cortical surface reconstruction method robust to inhomogeneities, we use AFCM instead of FCM to obtain fuzzy segmentations of the MR brain images into classes of GM, WM, and CSF.

We now briefly describe the steps of AFCM for 3-D images. Details of its derivation for 2-D images are provided in [9]. Let $y(\mathbf{x})$ be the observed image intensity at $\mathbf{x}, u_{k}(\mathbf{x})$ be the membership value at voxel $\mathbf{x}$ for class $k$ such that $u_{k}(\mathbf{x}) \geq 0$ and $\sum_{k=1}^{K} u_{k}(\mathbf{x})=$ $1, c_{k}$ be the centroid of class $k$, and $g(\mathbf{x})$ be the unknown gain field to be estimated. $K$, the total number of classes, is assumed to be 3. The steps for AFCM are as follows:

1. Provide initial values for centroids, $c_{k}, k=1, \ldots, K$, and set the gain field $g(\mathbf{x})$ equal to one for all $\mathbf{x}$.

2. Compute memberships as follows:

$$
u_{k}(\mathbf{x})=\frac{\left\|y(\mathbf{x})-g(\mathbf{x}) c_{k}\right\|^{-2}}{\sum_{l=1}^{K}\left\|y(\mathbf{x})-g(\mathbf{x}) c_{l}\right\|^{-2}}
$$


for all $\mathbf{x}$ and $k=1, \ldots, K$

3. Compute new centroids as follows:

$$
c_{k}=\frac{\sum_{\mathbf{x}} u_{k}^{2}(\mathbf{x}) g(\mathbf{x}) y(\mathbf{x})}{\sum_{\mathbf{x}} u_{k}^{2}(\mathbf{x}) g^{2}(\mathbf{x})}, k=1, \ldots, K
$$

4. Compute new gain field by solving the following space-varying difference equation for $g(\mathbf{x})$ :

$$
y(\mathbf{x}) \sum_{k=1}^{K} u_{k}^{2}(\mathbf{x}) c_{k}=g(\mathbf{x}) \sum_{k=1}^{K} u_{k}^{2}(\mathbf{x}) c_{k}^{2}+\lambda_{1}\left(g(\mathbf{x}) * H_{1}(\mathbf{x})\right)+\lambda_{2}\left(g(\mathbf{x}) * H_{2}(\mathbf{x})\right)
$$

where $H_{1}(\mathbf{x})=d_{x} * \check{d}_{x}+d_{y} * \check{d}_{y}+d_{z} * \check{d}_{z}$ and $H_{2}(\mathbf{x})=d_{x x} * \check{d}_{x x}+d_{y y} *$ $\check{d}_{y y}+d_{z z} * \check{d}_{z z}+2\left(d_{x y} * d_{x y}\right)+2\left(d_{y z} * d_{y z}\right)$. The operators $d_{x}, d_{y}, d_{z}$ are finite differences along the axes of the image volume, and $d_{x x}=d_{x} * d_{x}, d_{y y}=d_{y} * d_{y}$, $d_{z z}=d_{z} * d_{z}, d_{x y}=d_{x} * y$, and $d_{x z}=d_{x} * d_{z}$ are second order finite differences. Here we have used the notation $\breve{f}(i)=f(-i)$. The symbol ' $*$ ' denotes the discrete convolution operator.

5. If the algorithm has converged, then quit. Otherwise, go to Step 2.

Convergence is defined to be when the maximum change in the membership functions over all pixels between iterations is less than 0.01 . In Step 4 of the algorithm, $\lambda_{1}$ and $\lambda_{2}$ are parameters that control the expected smoothness of the inhomogeneity. These parameters were determined empirically, and have demonstrated robustness to inaccurate selection. The gain field $g(\mathbf{x})$ in Step 4 was computed using a multigrid algorithm (see [9]).

\subsection{Deformable Surface Model}

The method reported in [6] used a combination of traditional external forces and unconstrained pressure forces for the deformable surface model. The traditional external forces are computed by minimizing certain energy functions derived from the data $[7$, 8]. A deformable surface model using such external forces, however, has difficulties in progressing into boundary concavities [10]. Unconstrained pressure forces, proposed in $[11,8]$, can help push the deformable surface into boundary concavities. However, a deformable surface under unconstrained pressure forces can often behave arbitrarily and cause the surface to intersect itself. Recently, Xu and Prince [10] developed a new external force model, called gradient vector flow (GVF). The GVF external force field has a large capture range and is capable of forcing the deformable surface into boundary concavities. In this section, we give a brief overview of GVF used in this paper and describe our improved deformable surface model by using a combination of GVF and a constrained pressure force as its external forces.

The GVF field $\mathbf{v}(\mathbf{x})$ where $\mathbf{x}=(x, y, z) \in \mathbf{R}^{3}$ is defined as the equilibrium solution of the following system of partial differential equations

$$
\mathbf{v}_{t}=\mu \nabla^{2} \mathbf{v}-\left(\mathbf{v}-\nabla u_{\mathrm{gm}}\right)\left|\nabla u_{\mathrm{gm}}\right|^{2}
$$


where $\mathbf{v}_{t}$ denotes the partial derivative of $\mathbf{v}(\mathbf{x} ; t)$ with respect to $t$, and $\nabla^{2}=\frac{\partial^{2}}{\partial x^{2}}+$ $\frac{\partial^{2}}{\partial y^{2}}+\frac{\partial^{2}}{\partial z^{2}}$ is the Laplacian operator (applied to each spatial component of $\mathrm{v}$ separately), and $u_{\mathrm{gm}}$ is the GM membership function. When GVF is computed on thick edges, such as in the GM membership function, the resulting GVF field converges to the center of the thick edge.

We use a combination of GVF and a constrained pressure force to provide external forces for our deformable surface. The resulting external force is given by:

$$
\mathbf{F}_{\text {ext }}(\mathbf{x})=[<\mathbf{v}(\mathbf{x}), \mathbf{n}(\mathbf{x})>+C(\mathbf{x})] \mathbf{n}(\mathbf{x})
$$

where $\mathbf{v}(\mathbf{x})$ is the GVF field, $\mathbf{n}(\mathbf{x})$ is the outward normal vector of the surface at $x,\langle\cdot, \cdot\rangle$ is the inner product of two vectors, and $C(\mathbf{x})$ is a constraint field on the pressure force (defined below). Since the component of the external force in the tangent plane will only affect the parameterization of the surface but not its shape, we project $\mathbf{v}(\mathbf{x})$ onto the normal direction at surface position $\mathbf{x}$. Thus, the internal force is solely responsible for controlling the surface parameterization while the external force is solely responsible for deforming the surface towards the feature of interest.

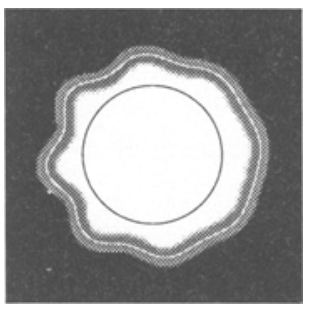

Fig. 2. Example of a deformable contour converging to the center of a simulated GM.

The constrained pressure force is similar in concept to the signed pressure force used in [12]. Unlike the unconstrained pressure force mentioned earlier, the constrained pressure force behaves more conservatively and is less subject to the self-intersection problem. It is used to increase the speed of convergence as well as reconstruction accuracy. The constraint field $C(\mathbf{x})$ is designed to turn off the pressure force once the surface enters the GM and leaves the surface under the influence of GVF external forces only. $C(\mathbf{x})$ is defined to be:

$$
C(\mathbf{x})= \begin{cases}0 & \text { if }\left|2 u_{\mathrm{wm}}(\mathbf{x})+u_{\mathrm{gm}}(\mathbf{x})-1\right|<\delta \\ 2 u_{\mathrm{wm}}(\mathbf{x})+u_{\mathrm{gm}}(\mathbf{x})-1 & \text { otherwise }\end{cases}
$$

where $u_{\mathrm{wm}}(\mathbf{x})$ and $u_{\mathrm{gm}}(\mathbf{x})$ are white matter and gray matter membership functions, and $\delta$ is a threshold to control the width of the gray matter region where the pressure force is disabled. $\delta$ is chosen to be 0.5 in our experiments. To help understand the behavior of the external forces, we apply a 2D deformable contour, a 2D analog to deformable surfaces in 3D, using external forces defined in (5) on a phantom simulating WM and GM of the brain. In Fig. 2, WM is depicted as the white region in the center and GM is depicted as the surrounding gray ribbon. The initial deformable contour is the circle shown in black and the final converged contour is shown in white. Note that the final contour indeed converges to the center of the simulated GM.

\section{Results}

The described cortex reconstruction method was applied to MR brain images from six subjects, four taken from the Baltimore Longitudinal Study on Aging [13]. Using an 
Table 1. Euler characteristics of surfaces generated for six subjects at different iterations.

\begin{tabular}{|c|c|c|c|c|c|c|c|c|c|c|c|c|}
\hline Iteration(s) & 0 & 1 & 2 & 3 & 4 & 5 & 6 & 7 & 8 & 9 & 15 & 20 \\
\hline Subject 1 & -757 & -49 & -6 & 2 & - & - & - & - & - & - & - & - \\
Subject 2 & -1010 & -56 & -14 & -4 & -2 & -2 & -2 & -2 & 0 & 2 & - & - \\
Subject 3 & -666 & -50 & -16 & -12 & -4 & -2 & 0 & 0 & 2 & - & - & - \\
Subject 4 & -860 & -66 & -24 & -8 & -6 & -2 & -2 & -2 & 2 & - & - & - \\
Subject 5 & -192 & -75 & -40 & -27 & -22 & -19 & -17 & -16 & -14 & -12 & -4 & 2 \\
Subject 6 & -462 & -26 & -12 & -6 & 0 & 2 & - & - & - & - & - & - \\
\hline
\end{tabular}

SGI O2 workstation with a $174 \mathrm{MHz}$ R10000 processor, the total processing time per subject varied between 4.5 and 6.5 hours. The time required for manual interaction varied between 0.5 hours and 1 hour for a trained operator. AFCM required approximately 1 hour. The automated steps in GM/WM interface estimation take about 0.5 hours which produces a mesh with between 200,000 and 400,000 vertices. Because of the large number of vertices in the mesh, it takes the deformable surface algorithm about 3 hours to produce the final reconstructed surface. Note that both AFCM and the deformable surface algorithm are fully automated steps.

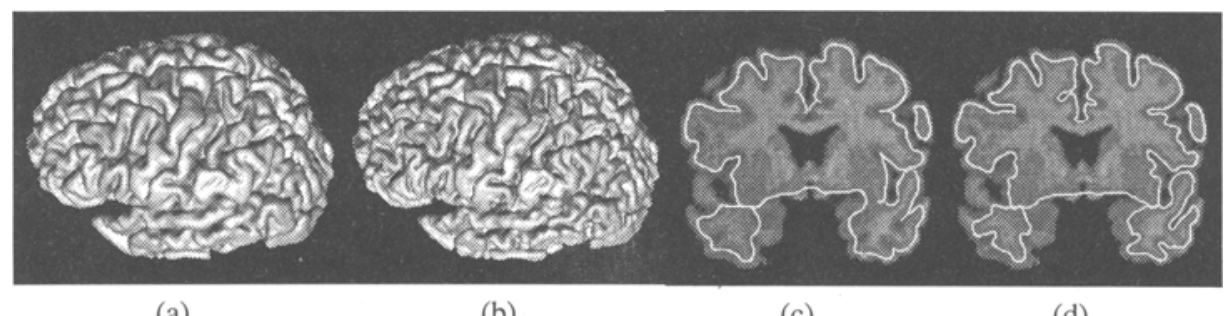

(a)

(b)

(c)

(d)

Fig. 3. Surface rendering of reconstructed cortical surface from one study using (a) the previous method, and (b) the current method. The coronal slice actoss the anterior commissure superimposed with the cross section of the corresponding reconstructed cortical surface using (c) the previous method, and (d) the new method.

The application of the method to multiple subjects allowed a preliminary validation of the convergence of the deformable surface initialization algorithm (see Sect. 2). In all six cases, the topology of the surface converged to the correct topology in less than 20 iterations. The result is shown in Table 1. Note that an Euler characteristic of 2 implies that the surface has a topology equivalent to a sphere and that a value smaller than 2 implies that the surface has one or more handles (like the handle of a coffee cup). The smaller the Euler characteristic is, the more handles a surface has. From the results, we observe that the median filter used in the iterative process effectively eliminated handles on the surface and that the Euler characteristic was nondecreasing with each iteration. 
In Figs. 3(a) and 3(b), we show the surface rendering of the reconstructed cortical surface from one subject using the previous and the new methods. Notice that although the two surfaces look similar overall, the new method seems to maintain more details. In Figs. 3(c) and 3(d), we show the coronal slice across the anterior commissure superimposed with the cross section of the corresponding reconstructed cortical surface using the previous and the new methods. It is apparent from this view that the new method yields more accurate results.

The accuracy of the previous and new methods were also compared quantitatively by computing landmark errors. A trained operator identified five landmarks on the central cortical layer for each hemisphere. The landmarks are located on the root of the central sulcus (CS), the crown of the post-central gyrus (PCG), the most anterior point of the temporal lobe (TL), midway along the calcarine fundus (CALC), and the medial frontal gyrus (MFG). The landmark error was then computed as the minimum distance between the given landmark and the surface. Table 2 shows the results of landmark errors using the previous and the new methods for two hemispheres. On average, the new method offers an improvement of approximately $25 \%$ over the previous method.

Table 2. Landmark errors comparison (in $\mathrm{mm}$ )

\begin{tabular}{|l|c|c|c|c|c|c|c|c|c|c||c|c|}
\hline Method & $\mathrm{CS}_{1}$ & $\mathrm{CS}_{2}$ & $\mathrm{PCG}_{1}$ & $\mathrm{PCG}_{2}$ & $\mathrm{TL}_{1}$ & $\mathrm{TL}_{2}$ & $\mathrm{CALC}_{1}$ & $\mathrm{CALC}_{2}$ & $\mathrm{MFG}_{1}$ & $\mathrm{MFG}_{2}$ & Mean & Std \\
\hline Previous & 1.9 & 2.6 & 0.7 & 0.7 & 0.5 & 1.3 & 1.0 & 0.4 & 0.9 & 1.0 & 1.10 & 0.68 \\
New & 0.5 & 2.1 & 0.7 & 0.4 & 0.5 & 1.4 & 0.7 & 0.4 & 0.5 & 1.1 & 0.83 & 0.55 \\
\hline
\end{tabular}

In Fig. 4, we show where the reconstructed cortical surfaces intersect coronal slices taken through the anterior commissures of each subject. These figures show that the surfaces reside on the central cortical layer and that buried gyri (such as the insula) are found. It should be noted that isolated closed 2-D contours appearing in these images are actually part of the 3-D surfaces, which are topologically equivalent to spheres. Although most gyri are properly modeled, certain regions, such as the superior temporal gyrus, are sometimes not found accurately. We are considering further improvements to correct these deficiencies and to further decrease the need for manual interaction.

\section{Acknowledgments}

The authors would like to thank Dr. R. Nick Bryan, Dr. Christos Davatzikos, and Marc Vaillant for their support in this work. They would also like to thank Dr. Ron Kikinis and Dr. Susan Resnick for providing MR data.

\section{References}

1. A. M. Dale and M. I. Sereno. Improved localization of cortical activity combining EEG and MEG with MRI cortical surface reconstruction: A linear approach. J. Cogn. Neuroscience, 5(2):162-176, 1993.

2. D. MacDonald, D. Avis, and A. C. Evans. Multiple surface identification and matching in magnetic resonance images. In SPIE Proc. VBC'94, volume 2359, pages 160-169, 1994. 


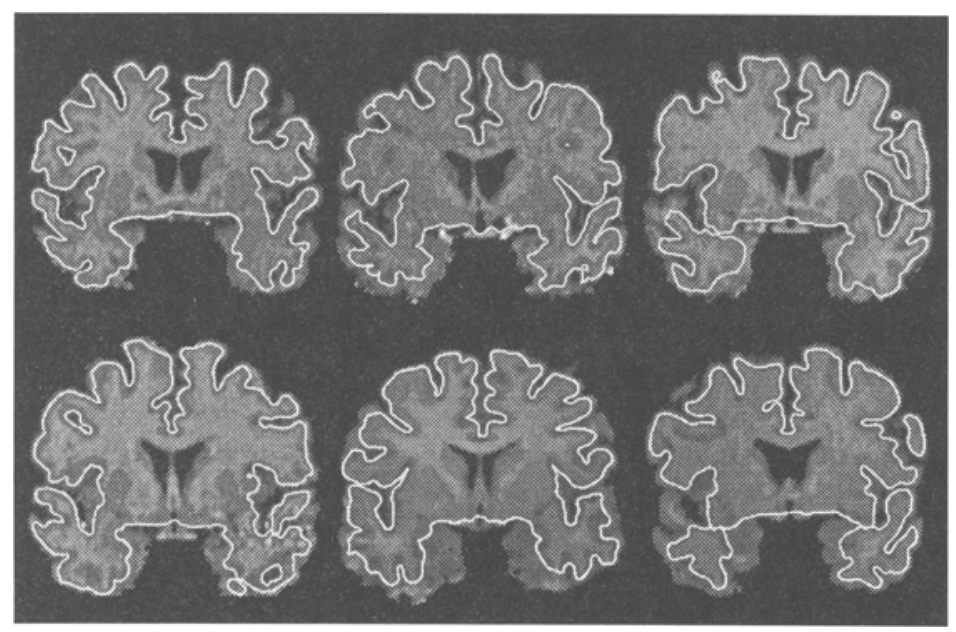

Fig. 4. From left to right and top to bottom, the coronal slice across the anterior commissure for subjects 1 to 6 superimposed with the cross section of the corresponding reconstructed cortical surface.

3. S. Sandor and R. Leahy. Towards automated labelling of the cerebral cortex using a deformable atlas. In Information Processing in Medical Imaging, pages 127-138, 1995.

4. C. Davatzikos and R. N. Bryan. Using a deformable surface model to obtain a shape representation of the cortex. IEEE Trans. Med. Imag., 15:785-795, December 1996.

5. H.A. Drury, D.C. Van Essen, C.H. Anderson, C.W. Lee, T.A. Coogan, and J.W. Lewis. Computerized mappings of the cerebral cortex: A multiresolution flattening method and a surfacebased coordinate system. J. Cogn. Neuroscience, pages 1-28, 1996.

6. C. Xu, D. L. Pham, and J. L. Prince. Finding the brain cortex using fuzzy segmentation, isosurfaces, and deformable surface models. In the XVth Int. Conf. Inf. Proc. Med. Imag. (IPMI), pages 399-404. Springer-Verlag, 1997.

7. M. Kass, A. Witkin, and D. Terzopoulos. Snakes: Active contour models. International Journal of Computer Vision, 1(4):321-331, 1987.

8. L. D. Cohen and I. Cohen. Finite-element methods for active contour models and balloons for 2-D and 3-D images. IEEE Trans. on Pattern Anal. Machine Intell., 15(11):1131-1147, November 1993.

9. D. L. Pham and J. L. Prince. An adaptive fuzzy c-means algorithm for image segmentation in the presence of intensity inhomogeneities. In SPIE Medical Imaging '98: Image Processing. SPIE, Feb. 21-27, 1998. to appear.

10. C. Xu and J. L. Prince. Snakes, shapes, and gradient vector flow. IEEE Trans. on Image Processing, pages 359-369, March 1998.

11. L. D. Cohen. On active contour models and balloons. CVGIP: Image Understanding, 53(2):211-218, March 1991.

12. T. Kapur, E. Grimson, W. Wells, and R. Kikinis. Segmentation of brain tissue from magnetic resonance images. Medical Image Analysis, 1(2):109-127, 1996.

13. N. W. Shock, R. C. Greulich, R. Andres, D. Arenberg, P. T. Costa Jr., E. Lakatta, and J. D. Tobin. Normal human aging: The Baltimore longitudinal study of aging. U.S. Governement Printing Office, Washington, D.C., 1984. 